\section{Comparison of medium to long- term outcomes of acute severe ulcerative colitis patients receiving accelerated and standard infliximab induction}

Accelerated dose infliximab (IFX) induction is associated with a reduced short-term colectomy rate in acute severe ulcerative colitis (ASUC). In this issue Gibson et al report the medium and long term outcome - 145 patients, four centres, comparing standard $(0,2$, 6 weeks) with accelerated dose ( 3 doses by 4 weeks) induction (See page 441). In the participating centres Accelerated Dose induction (AD) has been used since 2014. Standard dose induction (SD) was classified by time, SD1 before and SD2 after 2014. Disease severity at induction was comparable in SD1 and AD groups but less in SD2. Time to colectomy was prolonged in $\mathrm{AD}$ and SD2 compared with SD1 - see figure two. In a Cox regression analysis factors independently associated with increased risk of colectomy included an endoscopic Mayo score of 3 at induction (HR 2.2 (95\% CI 1.13 to 4.28$), \mathrm{p}=0.021$ ) and increased CRP:ALB ratio at induction (HR 1.17 (95\% CI 1.04 to 1.32), $\mathrm{p}=0.011$ ). Conversely, use of an accelerated IFX induction regime was the only protective factor (HR 0.68 (95\% CI 0.47 to 0.97$), p=0.038$ ). In summary therefore since 2014 with stratified use of IFX, $\mathrm{AD}$ induction in the more severe patients, outcome is improved. This data further supports the use of $\mathrm{AD}$ induction in selected patients with ASUC.

There is an excellent accompanying Leading Article - Infliximab in acute severe colitis: getting the right dose (See page 427).

\section{Diagnostic ileocolonoscopy: getting the basics right}

Colonoscopy is a core skill and requires patience, practise and a logical approach to problem-solving. Caecal intubation and adenoma detection rates (ADRs) are quality benchmarks achieved by technique that optimises patient comfort while enhancing pathology detection and subsequent therapy. In this issue Choy et al, in an excellent practice-based review, discuss 'getting the basics right' as part of the pathway to achieving this. This includes comprehensive sections on preparation for endoscopy, scope handling, position changes, abdominal pressure and dealing with loops with helpful figures illustrating just how may different loops you can create and what are trying to achieve with position changes and abdominal pressure. Essential reading for trainees and a useful update for the experienced endoscopist. Editor's choice this month (See page 484).

\section{Variation in exposure to endoscopic haemostasis for acute upper gastrointestinal bleeding during UK gastroenterology training}

Gastroenterologists are generally expected to be competent in endoscopic haemostasis for acute upper gastrointestinal bleeding (AUGIB), with the Certificate of Completion of Training (CCT) often heralding the onset of participation in on-call AUGIB rotas. In this issue Siau et al report the volume of experience prior to award of CCT recorded on the Joint Advisory Group on Gastrointestinal Training System (JETS) e-portfolio for a cohort of 232 newly appointed consultants (See page 436). Median number of haemostasis procedures recorded were 42 (IQR 21-71). Experience of exposure to nonvariceal modalities (median 28, IQR 15-52) was more frequent than variceal therapies (median 11, IQR 5-22; $\mathrm{p}<0.001)$. By procedure, epinephrine injection (median 12, IQR 6-23) and variceal band ligation (median 10, IQR 5-20) were most commonly recorded, whereas sclerotherapy experience was rare (median 0, IQR 0-1). In separate article Segal and colleagues' have surveyed trainees on their experience - Training in endotherapy for acute upper gastrointestinal bleeding: a UKwide gastroenterology trainee survey (See page 430); 181 Trainees, 33.5\% response rate. There was significant variation in training with, for example, senior trainees reporting a lack of experience with independently applying glue (86\%), haemospray (54\%), heater probe $(36 \%)$ and variceal banding (36\%). It is clear from these data, and particularly with the advent of shape of training, it will be important to increase the opportunities for trainee exposure and practice (including through simulation based training). In addition endoscopy departments will need to ensure the availability of supportive provisions in haemostasis (ie, training/upskilling, supervision, mentorship) during the early post-CCT period.

There is an excellent linked commentary by Ian Penman - Training in endoscopic haemostasis: targeting the bleeding point (See page 425).

\section{Acute- on- chronic liver failure: definition, prognosis, and management}

Acute on chronic liver failure (ACLF) is a recently described (2002) entity in chronic liver disease - helpful to the clinician - and defined by acute hepatic decompensation with organ failure and a high short term risk of mortality. It occurs in $20 \%-30 \%$ of hospitalised patients with cirrhosis. In this edition Amin et al review the definition, management and prognosis. The condition is distinct from acute liver failure and stable progression of cirrhosis. Alcohol (acute or chronic) and infections are common precipitating factors. Prevention and early treatment of organ failure are key to survival. Outcome of liver transplantation - if available - is generally good. The review is an excellent update with ACLF being a significant challenge, resource heavy and the subject of a significant amount of ongoing research (See page 458).

\section{Non- alcoholic fatty liver disease (NAFLD) in non- obese individuals}

Non- alcoholic fatty liver disease (NAFLD) occurs in non- obese individuals $(\mathrm{BMI}<25)$ who can go on to develop Non-alcoholic steatohepatitis and progress to end stage liver disease. The pathophysiology is not fully understood relating to genetic factors, body composition and insulin resistance. The potential for lifestyle intervention is less clear than with the obese patient. In this issue Phipps et al review, in detail, the clinical features, pathophysiology, risk factors and highlight areas for future 


\section{UPFRONT}

research. It is interesting for example that when compared with lean patients without NAFLD, those with NAFLD have higher fasting glucose, increased BMI, increased waist circumference, and decreased circulating serum adiponectin levels, suggesting that lean and non- lean NAFLD patients share a common altered metabolic profile. It is essential that the non-obese patients with NAFLD are included in clinicals trials to impact on outcome with phase two and phase three trials underway investigating agents that target glucose and lipid metabolism, inflammation, and fibrosis.

This excellent update is clear and focused and great CPD for both the generalist and specialist (See page 478).

\section{Finally}

Please enjoy this issue. Please continue to read, enjoy, and feedback on the journal. Follow us on twitter @FrontGastro_BMJ and listen to our regular podcasts accessed via the journal website https://fg.bmj. $\operatorname{com} /$.

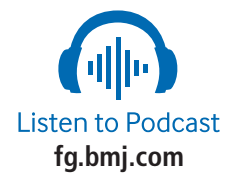

ORCID iD

R Mark Beattie http://orcid.org/ 00000003-4721-0577 